\title{
Improved regularized phase-tracking technique for the processing of squared-grating deflectograms
}

\author{
Jesús Villa, Juan Antonio Quiroga, and Manuel Servín
}

\begin{abstract}
We propose a robust procedure based on the regularized phase-tracking (RPT) technique to demodulate squared-grating deflectograms. The use of squared gratings, already reported, lets us multiplex the information of the deflections in two orthogonal directions in a single image, thus avoiding the necessity of rotating the gratings. The good noise-rejection characteristics of the RPT technique are improved by use of a quasi-Newton optimization algorithm and a quality-map-based algorithm for the crystal-growing process. (C) 2000 Optical Society of America
\end{abstract}

OCIS codes: $\quad 120.2650,120.4120,100.2650,170.4460,100.5070,120.5050$.

\section{Introduction}

Moiré deflectometry is a widely known technique for measuring ray-deflection maps caused by phase objects, based on moiré and Talbot effects. ${ }^{1,2}$ The applications of the technique range from reflectivesurface analysis ${ }^{3}$ to the measurement of refractive index and temperature distribution. ${ }^{4,5}$ In our particular case the application objective is to determine the power distribution in ophthalmic lenses. The experimental setup we used is shown in Fig. 1. A collimated beam crosses the phase object to be tested followed by a pair of linear transmission gratings G1 and G2 placed at a Talbot distance $\mathrm{Z}$ from each other. The Talbot image of G1 in the plane of G2 is deformed, owing to ray deflections caused by the object under test. The deflection information is codified in the resulting distorted moiré fringe pattern, which is observed in screen $\mathrm{S}$ by a CCD camera.

Several techniques have been applied to retrieve deflection information from deflectograms. Servín and Rodriguez-Vera ${ }^{6}$ measured the deviation of the fringe with respect to the unperturbed moiré fringe. In this way the deflection in the direction perpendicular to the reference fringes is measured. Canabal

J. Villa (jvilla@andromeda.cio.mx) and M. Servín are with the Centro de Investigaciones en Óptica, León, Gto., México. Apartado Postal 1-948, C. P. 37000. J. A. Quiroga is with the Departamento de Óptica, Facultad de Ciencias Físicas, Universidad Complutense de Madrid, C. P. 28040, Madrid, Spain.

Received 7 May 1999; revised manuscript received 22 September 1999.

0003-6935/00/040502-07\$15.00/0

(C) 2000 Optical Society of America and Quiroga ${ }^{7}$ proposed the measurement of local fringe direction to compute the local power of ophthalmic lenses. These techniques are based on either fringe maximum intensity location or gradient computation; thus they are more sensitive to noise.

Other possibilities are the phase-measurement methods. In moiré deflectometry the phase associated to the fringe pattern is directly related to the deflection at every point. The phase-shifting technique was applied by Pfeifer and Wang ${ }^{8}$ and by Canabal et al. ${ }^{9}$ The necessary phase shifts are obtained by lateral movement of one grating with respect to the other. The main problems with using Ronchi rulings are the diffraction effects and the assumption that the fringe pattern is sinusoidal. Studies to minimize these problems were reported by Keren and Kafri ${ }^{10}$ and by Bar-Ziv. ${ }^{11}$

An additional method is the Fourier-transform technique proposed by Takeda et al. ${ }^{12}$ This technique solves the problems associated with higher harmonics produced by Ronchi rulings. An early application of the Fourier transform in Talbot interferometry can be found in Ref. 13 .

If linear gratings are used, all these techniques need at least two images rotated $90^{\circ}$ from each other to obtain the complete deflection information. Quiroga et al. ${ }^{14}$ proposed the Fourier-transform method, using squared gratings to multiplex the complete information of deflections in a single image, thus avoiding the requirements mentioned above.

Despite its advantages, the Fourier-transform method still has some problems. First, the boundaries of the area of interest are an important error source. This problem can be solved by use of the Gerchberg extrapolation algorithm. ${ }^{15}$ Second, the 


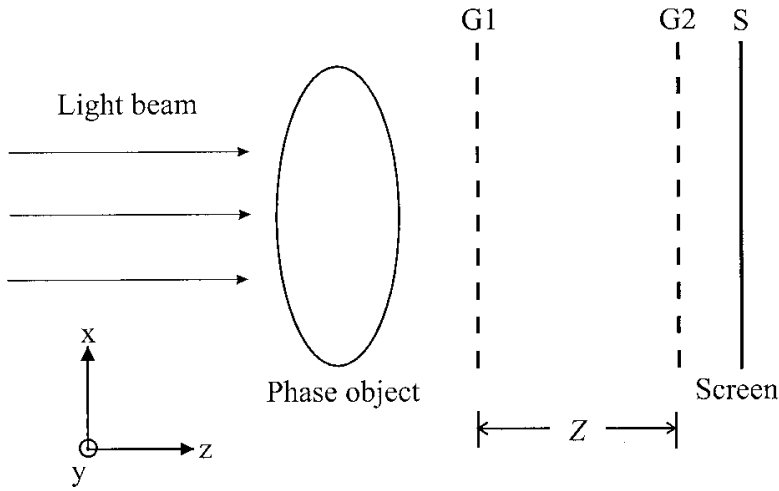

Fig. 1. Typical experimental setup used in moiré deflectometry. Gratings G1 and G2 are placed at a Talbot distance Z from each other to form a moiré fringe pattern. The fringe image is observed in the screen $\mathrm{S}$.

Fourier-transform technique assumes the carrier to be constant. Iterative procedures can be used to minimize this problem. ${ }^{16}$ Finally, the sidelobes containing the deflection information must be isolated by a bandpass filter of a size determined by the bandwidth of the signal. If we want to recover the complete information contained in the sidelobe, we cannot avoid that the noise present in this area is retrieved as well. This problem may be solved if we use a narrow bandpass filter, but this implies that the higher frequencies of the signal will be lost. The noise present in the recovered phase can be a drawback if gradient information must be computed from the estimated phase, as is the case in the measurement of power distribution of ophthalmic lenses. ${ }^{14}$

In this study the regularized phase-tracking (RPT) technique ${ }^{17}$ is applied to retrieve the continuous deflection and its gradients from deflectograms obtained with squared gratings. A squared grating can be considered to be the superposition of two linear Ronchi gratings with an angle of $90^{\circ}$ between them. The use of squared gratings for the simultaneous acquisition of two independent pieces of information is a known procedure in shearing interferometry 18 and experimental mechanics. ${ }^{19}$ In our case the use of these kinds of grids enables the processing of two orthogonal ray deflections.

As we show below, RPT can solve the above-mentioned problems of the Fourier-transform method while keeping its good properties. The principle of the method together with practical considerations regarding the use of squared gratings are presented in Section 2. Experimental results obtained with a progressive addition lens as well as comparison with measurements made with a commercial focimeter are presented in Section 3. Finally, conclusions are given in Section 4.

\section{Application of Regularized Phase Tracking to Squared-Grating Deflectograms}

\section{A. Regularized Phase-Tracking Technique}

The phase estimation of a fringe pattern may be formulated as an inverse problem that must be solved by an algorithm that incorporates a prior smoothness constraint about the phase being detected. To solve this problem, we may regularize it by proposing a suitable cost function with two terms related to the following: (1) the fidelity between the estimated function and the observations and (2) the smoothness of the phase being detected. It is assumed that the estimated phase function is the minimizer of the proposed cost function.

In the RPT technique developed by Servín et al. ${ }^{17}$ the fringe pattern is considered to be locally monochromatic; that is, the local irradiance is modeled as a cosinusoidal function phase modulated by a plane. The modeled cosinusoidal function must be close to the irradiance of the pattern that corresponds to the fidelity term. In this way the phase must be adapted to every region in the pattern. Smoothness and continuity of the estimated phase are enforced by the regularization term. The cost function proposed by Servín et al. ${ }^{17}$ is expressed as

$$
U_{T}=\sum_{(x, y) \in L} U_{x, y}\left(\phi, \omega_{x}, \omega_{y}\right)
$$

where

$$
\begin{aligned}
U_{x, y}\left(\phi, \omega_{x}, \omega_{y}\right)= & \sum_{(\tilde{x}, \tilde{y}) \in\left(N_{x, y} \cap L\right)}\left\{\mid g_{h}(\tilde{x}, \tilde{y})-\cos \left[\phi_{e}(x, y, \tilde{x}, \tilde{y})\right.\right. \\
& \left.+u_{0} x+v_{0} y\right]\left.\right|^{2}+\lambda \mid \phi(\tilde{x}, \tilde{y}) \\
& \left.-\left.\phi_{e}(x, y, \tilde{x}, \tilde{y})\right|^{2} m(\tilde{x}, \tilde{y})\right\} \\
\phi_{e}(x, y, \tilde{x}, \tilde{y})= & \phi(x, y)+\omega_{x}(x, y)(x-\tilde{x}) \\
& +\omega_{y}(x, y)(y-\tilde{y})
\end{aligned}
$$

where $U_{x, y}$ is the energy of the system at a site $(x, y)$ in the image. $L$ is a two-dimensional lattice that has valid data, and $N_{x, y}$ is a neighborhood region around the coordinate $(x, y)$ in the image where the phase is being detected. The field $m(x, y)$ is an indicator that equals 1 if the site has already been estimated and is 0 otherwise. The fields $\omega_{x}$ and $\omega_{y}$ are the estimated local frequencies in the $x$ and the $y$ directions, which are the first derivatives of the phase; in this way no explicit differentiation over the estimated phase is required. The possible presence of a carrier frequency is indicated by $u_{\mathrm{o}}$ and $v_{\mathrm{o}}$, which stand for the components of the carrier frequency. The so-called regularizing parameter $\lambda$ controls the smoothness of the detected phase. The choice of this parameter has been widely discussed in the literature, and no precise formula can be applied to determine an optimal value for a given problem so that it is commonly selected intuitively (see, for example, Ref. 20). The field $g_{h}(x, y)$ is a high-pass-filtered and normalized version of the fringe pattern. As Servín et al. ${ }^{17}$ mentioned, function (1) is multimodal; so it may have several minima. Thus the minimization of this function is a difficult task. Following Servin et al. ${ }^{17}$ we compute a first estimation of the phase by minimizing local cost function (2) with the so-called crystal-growing algorithm. 
In the frequency domain we may consider the RPT to be a narrow bandpass adaptive filter that is moving smoothly with high inertia over the spectrum of the signal. This characteristic presents the additional advantage of a large gain in the signal-to-noise ratio of the recovered signal.

\section{B. Considerations for Use of Regularized Phase Tracking} with Squared-Grating Deflectograms

When squared gratings are used in a deflectometer such as the one depicted in Fig. 1, a fringe pattern with two fringe systems containing the complete deflection information is obtained. ${ }^{14}$ If we use Ronchi gratings, the model for this fringe pattern image may be expressed as

$$
g(x, y)=\sum_{n=-\infty}^{\infty} a_{n}{ }^{2} \cos \left[n \Phi_{x}(x, y)\right] \sum_{m=-\infty}^{\infty} \cos \left[m q \Phi_{y}(x, y)\right],
$$

where

$$
\begin{aligned}
& \Phi_{x}(x, y)=Z q \phi_{x}(x, y)+u_{0} x+v_{0} y, \\
& \Phi_{y}(x, y)=Z q \phi_{y}(x, y)+v_{0} x-u_{0} y,
\end{aligned}
$$

with $u_{\mathrm{o}}=q(\cos \theta-1)$ and $v_{\mathrm{o}}=q \sin \theta$, where $Z$ is the distance between gratings, $q=2 \pi / p$, $p$ is the period of the grating, $\theta$ is the angle between the gratings, and $\phi_{x}$ and $\phi_{y}$ are the deflections we are looking for. In a simplified form we can rewrite Eq. (4) as

$$
g_{h}(x, y)=\cos \left[\Phi_{x}(x, y)\right] \cos \left[\Phi_{y}(x, y)\right],
$$

where we have neglected higher harmonics and assumed that no dc term is present (for a Ronchi ruling, $a_{n}$ is proportional to $1 / n^{2}$ ).

The selection of the component to be processed is by means of the carrier frequency. As with the Fourier method, the carrier lets us differentiate between $\phi_{x}$ and $\phi_{y}$. For example, if we choose as a carrier $u_{0} x+$ $v_{\mathrm{o}} y$ from minimizing Eq. (2) with $g_{h}(x, y)$ given by Eq. (7), we should obtain three fields corresponding to $\phi_{x}$, $\partial \phi_{x} / \partial x$, and $\partial \phi_{x} / \partial y$.

The model we are using in the RPT (a single cosinusoidal function) differs from the model of the observed data (two crossed cosinusoidal functions). In this way when we select one of the fringe patterns by means of the carrier we are treating the other as interfering noise that might introduce errors into the phase estimation. Servin et al. ${ }^{17}$ proposed the use of the gradient-descent method to minimize function (2). With squared gratings this simple algorithm is not robust enough, even when the two deflections are clearly separated in the frequency domain.

Taking into account the results obtained, we decided to use a more efficient algorithm to minimize cost function (2). The selected minimizing algorithm was the Broyden-Fletcher-Goldfarb-Shanno; this is a quasi-Newton-type method. We tested this algorithm with real images, obtaining good results. However, the cost of the robustness of the optimization algorithm is a greater processing time compared with that proposed in Ref. 17 (this is discussed in
Section 3 below). The implementation of this algorithm is easy, since it is already included as part of the optimization toolbox of the MATLAB environment. ${ }^{21}$

In the RPT technique the phase estimation is performed as a growing process. Local function (2) is minimized point by point by means of the algorithm mentioned above. In general, minimization algorithms require good initial values in order to reach the correct solution. Thus, once one pixel is processed, the obtained solution $\left[\phi, \omega_{x}, \omega_{y}\right]$ at pixel $(x, y)$ is used as the initial value in the minimization of local cost function (2) at the next neighbor pixel (the problem of the starting pixel is addressed below). This procedure is path dependent, because the estimation depends on adjacent sites that have already been estimated; hence the path selection for the crystal-growing process becomes important with noisy fringe patterns. The usual way to perform the crystal-growing process is simply by scanning row by row. This procedure does not take into account the local quality of the fringe pattern. In the case of ophthalmic lenses the quality of the fringe pattern in the borders of the area of interest is poor. Also, in the areas of high power there exists an increase of the fringe density and therefore a modulation decrease that is due to the discrete sampling with finite size elements. For these reasons the crystal-growing algorithm must be able to select the path that processes the highest-quality areas first.

For this purpose the quality-map-based algorithm of Ströbel ${ }^{22}$ is applied for the crystal-growing process. The quality map used by Ströbel ${ }^{22}$ is the amplitude modulation of the fringe pattern calculated by standard phase-shifting techniques. In our case we are analyzing single fringe patterns with a carrier; that is, there is no possibility to compute the amplitude (quality) map with phase shifting. However, we can apply the Fourier-transform technique and compute the amplitude map of the resulting complex signal. We obtained the best results by quantifying the quality map to a few values, typically 4 or 5 . As mentioned, one of the drawbacks of the Fourier-transform method is the poor performance in the borders of the area of interest. That is, the computed quality map is not reliable in such borders. For this reason, in addition to the quantification, the zones adjacent to the borders are set to the lowest-quality value. In this way the borders are processed last.

With the RPT guided by a quality map, the demodulation starts from the highest-quality pixel; however, since no pixel has been processed, no initial values for the minimization of local cost function (2) are available at this site. A drawback of the quasiNewton optimization methods is that they require a sufficiently precise initial approximation of the solution to ensure the convergence. ${ }^{23}$ This is a problem, because in the RPT technique the estimation at the first point is important for avoiding further error propagation. In fact, with squared-grating deflectograms the demodulation of the starting point determines the failure or the success of the demodulation 


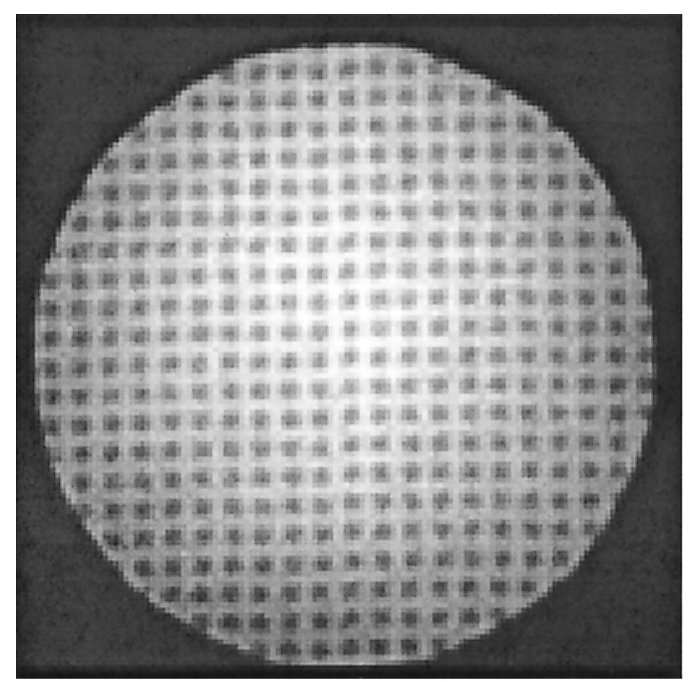

(a)

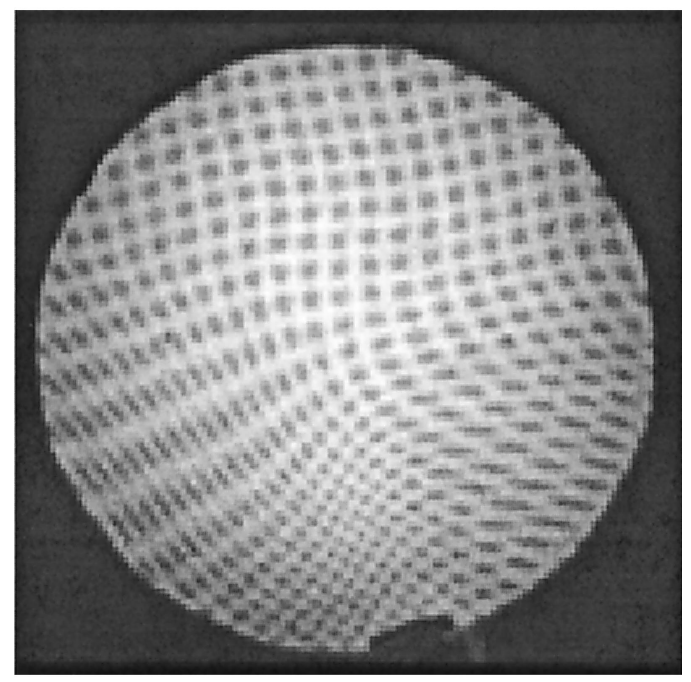

(b)

Fig. 2. (a) Reference and (b) distorted moiré deflectograms for a progressive addition lens obtained with squared gratings.

of the whole fringe pattern. For this reason the stimation at the first pixel is performed with the simple gradient-descent method to ensure a close approximation to the solution.

\section{Experimental Results}

To test the proposed procedure to demodulate squared-grating deflectograms, we measured the spherical and the cylindrical power of a progressive addition lens, in particular, a left-eye Hoyalux progressive lens. The results obtained were compared with the measurements made with a commercial focimeter. The reference deflectogram and the distorted deflectogram are shown in Figs. 2(a) and 2(b), respectively. The size of the images is $128 \times 128$ pixels with 256 gray levels. To demodulate each of them, we determined from the reference deflectogram the values of the fringe carrier frequencies. The recovered phase from the reference is subtracted

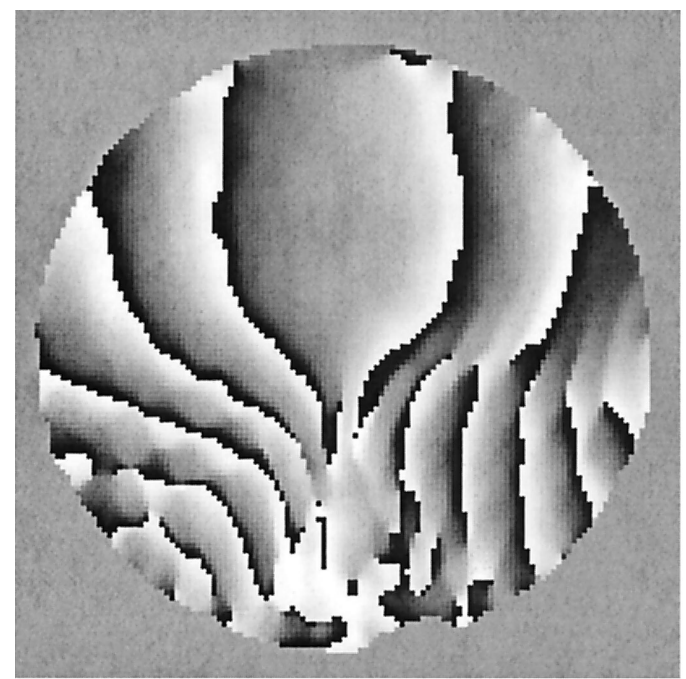

(a)

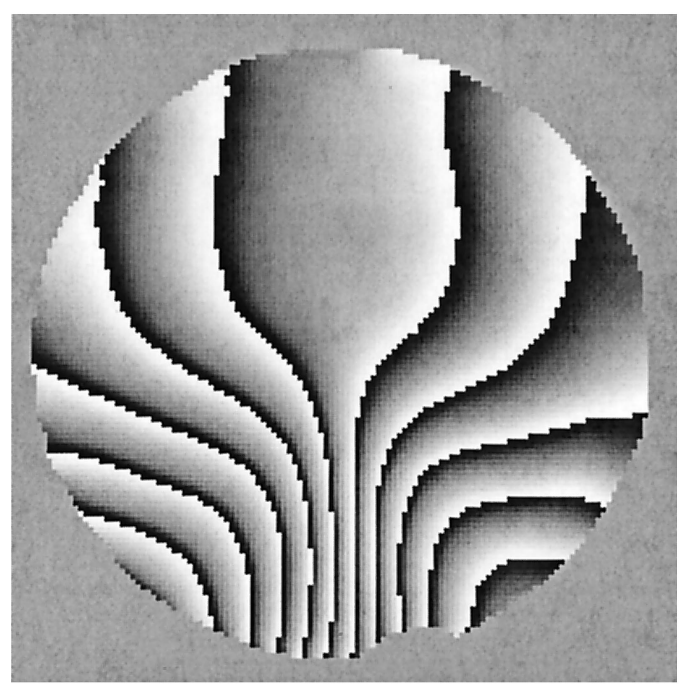

(b)

Fig. 3. Phase map corresponding to the deflection in the $x$ direction obtained with (a) the Fourier method and (b) the RPT technique.

from the phase of the distorted deflectogram to correct the error in the determination of the carrier frequencies.

A comparative experiment was made to process the deflectogram shown in Fig. 2(b). Figure 3(a) shows the deflection in the $x$ direction obtained with the Fourier method by use of a Gaussian filter. Figure 3(b) shows the same deflection obtained with RPT. As can be seen in Fig. 3(a), the Fourier-transform method cannot recover the deflection information, especially in the high-frequency areas; this is because the carrier is not high enough to separate completely the sidelobe information by means of a simple bandpass filter. As we explained above, the RPT behaves like an adaptive narrow bandpass filter that allows for complete recovery of the signal with a high signalto-noise ratio. In the case of measuring power dis- 


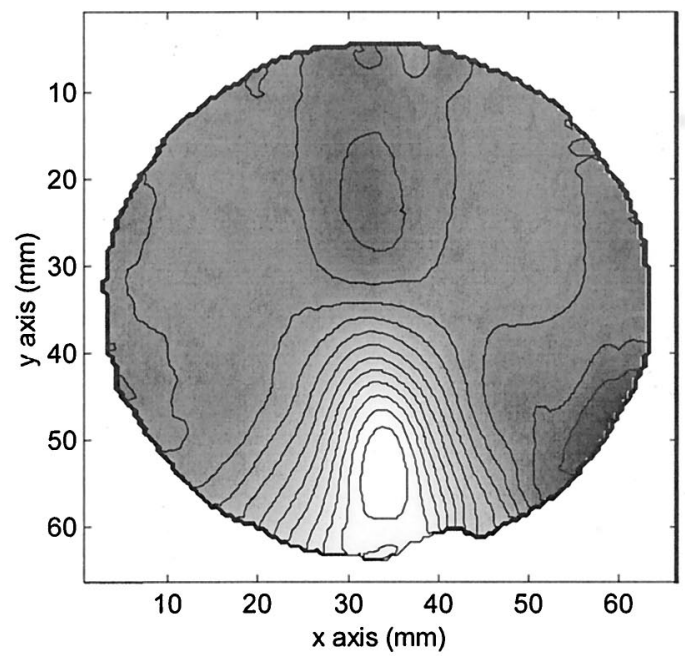

(a)

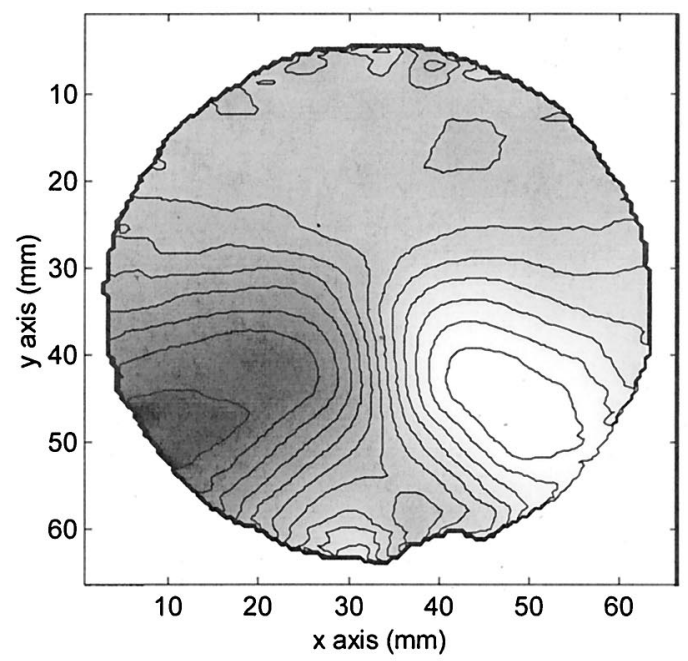

(b)
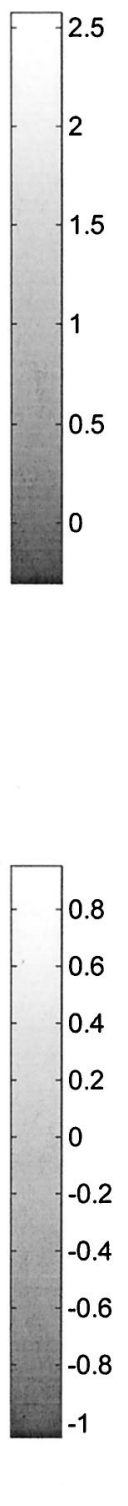

Fig. 4. Contour maps (scaled in dioptries) corresponding to (a) $\omega_{x x}$, (b) $\omega_{x y}$, (c) $\omega_{y x}$, and (d) $\omega_{y y}$.

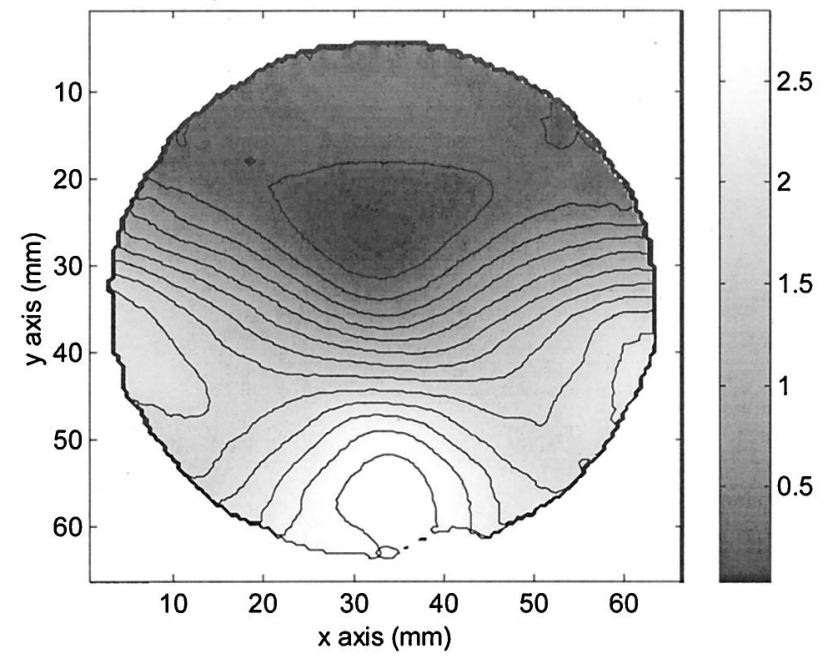

(c)

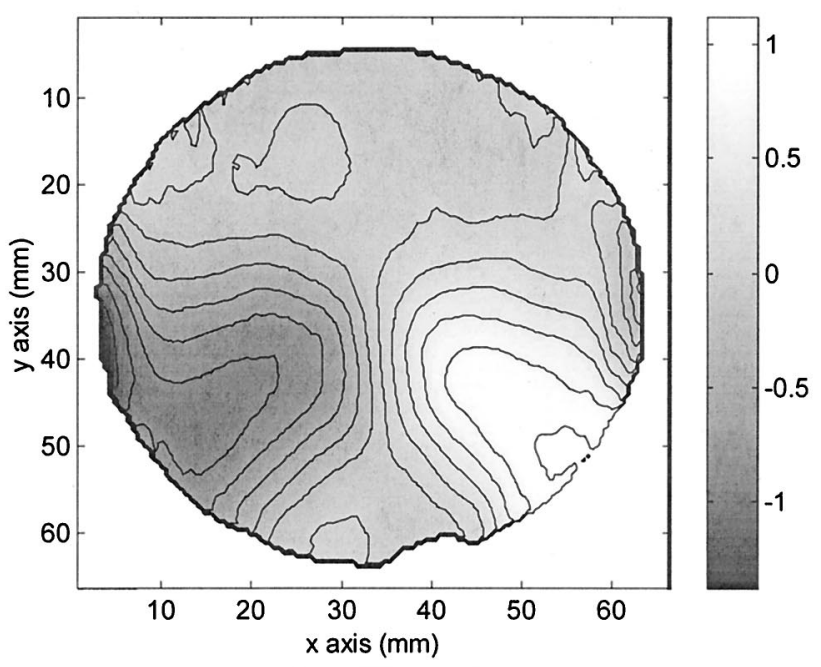

(d) tributions in lenses, the problem of using the Fourier method becomes even worse because of the necessity of computing the gradients of the obtained deflection map.

In the particular case of ophthalmic lenses the interesting parameters are the spherical and the cylindrical powers that can be computed from the gradients of the deflection components. As mentioned above, the frequency fields $\omega_{x}$ and $\omega_{y}$ of cost function (2) represent the derivatives of the deflection to be processed. We rename frequency fields $\omega_{x}$ and $\omega_{y}$ of Eq. (3) as $\omega_{x x}$ and $\omega_{x y}$ when we compute the $x$ component of the deflection, and we rename $\omega_{x}$ and $\omega_{y}$ when we compute the $y$ component; that is, they represent the derivative of $\phi_{x}$ without explicit differentiation. The same is true for the frequency fields $\omega_{y x}$ and $\omega_{y y}$ computed together with the $y$ component of the deflection $\left(\phi_{y}\right)$; that is

$$
\omega_{x x}=\frac{\partial \phi_{x}}{\partial x}, \quad \omega_{x y}=\frac{\partial \phi_{x}}{\partial y}, \quad \omega_{y y}=\frac{\partial \phi_{y}}{\partial y}, \quad \omega_{y x}=\frac{\partial \phi_{y}}{\partial x} .
$$

From the fields $\omega_{x x}, \omega_{x y}, \omega_{y y}$, and $\omega_{y x}$ we can compute the cylindrical and the spherical powers $\mathrm{as}^{24}$

$$
\begin{aligned}
& C=\left[\left(\omega_{x x}+\omega_{y y}\right)^{2}-4\left(\omega_{x x} \omega_{y y}-\omega_{x y} \omega_{y x}\right)\right]^{1 / 2}, \\
& S=1 / 2\left(\omega_{x x}+\omega_{y y}-C\right),
\end{aligned}
$$

respectively. Figures 4(a), 4(b), 4(c), and 4(d) show the contour maps (scaled in dioptries) of $\omega_{x}, \omega_{x y}, \omega_{y y}$, 


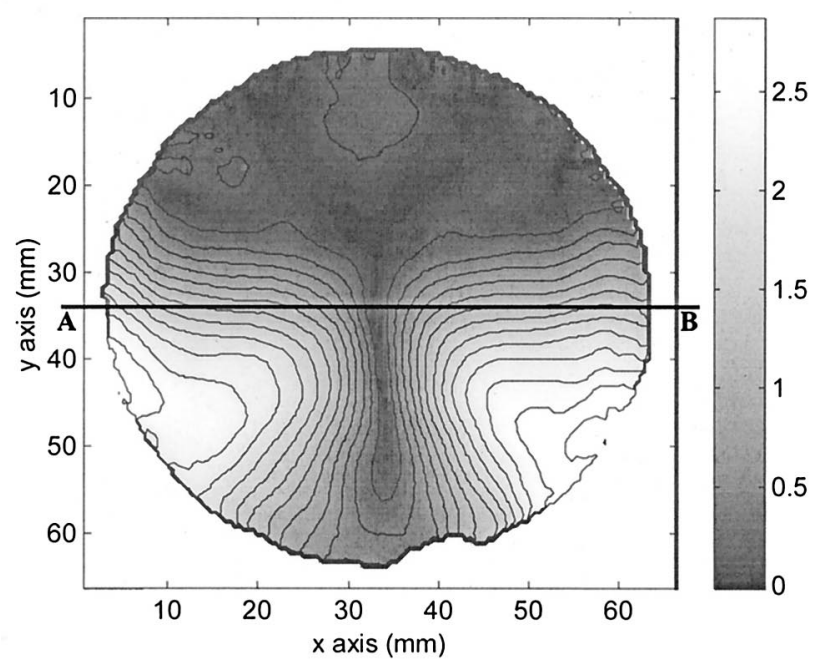

(a)

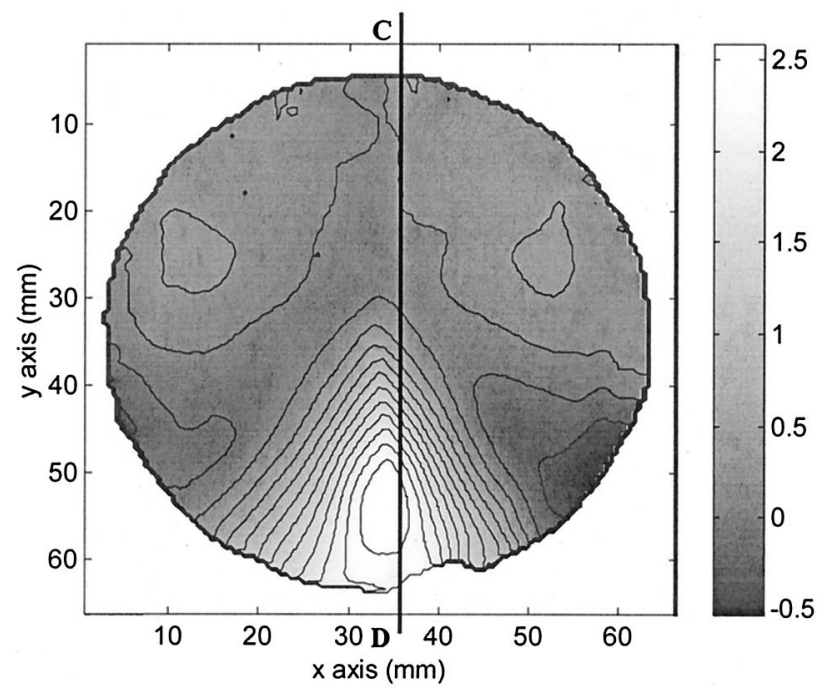

(b)

Fig. 5. (a) Cylindrical and (b) spherical power computed with the RPT technique.

and $\omega_{y x}$, respectively. The employed value of the regularization parameter $\lambda$ of Eq. (2) was 5 in all cases. The neighborhood size $N_{x y}$ was $11 \times 11$ pixels. As mentioned above, the use of a more sophisticated optimization algorithm implies a greater processing time compared with the 2 min taken with the gradient descent.17 In our case the processing time was 20 min for each deflection in a AMD K6-2 $330-\mathrm{MHz}$ computer equipped with 128 Mbytes of RAM. The contour maps of the cylindrical and the spherical powers are shown in Figs. 5(a) and 5(b), respectively Figures $6(\mathrm{a})$ and $6(\mathrm{~b})$ show the profiles of the cylinder and the sphere along lines $A B$ and $C D$ of Figs. 5(a) and 5(b), respectively, together with the measurement made with a commercial focimeter

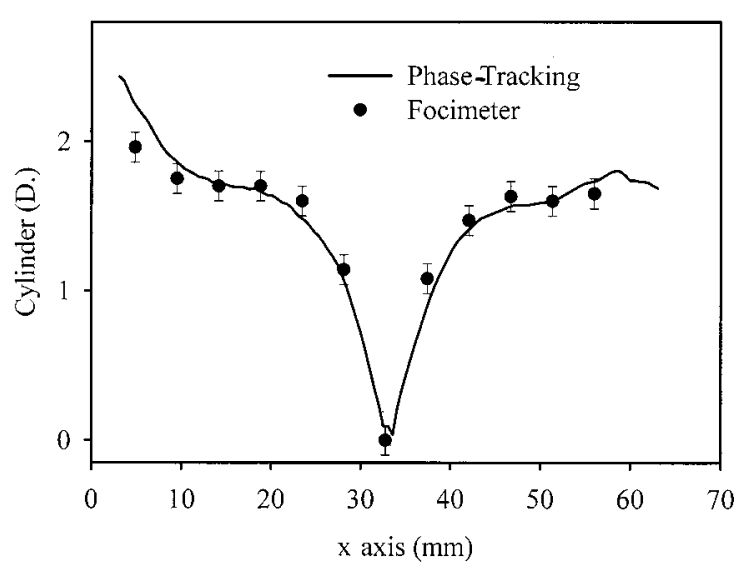

(a)

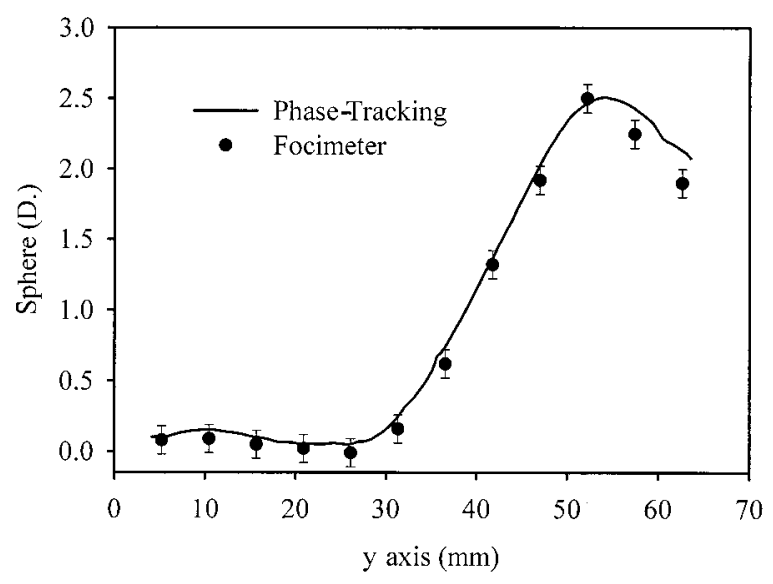

(b)

Fig. 6. Profiles of (a) cylindrical power along line $A B$ in Fig. 6(a) and (b) spherical power along line $C D$ in Fig. 6(b), together with the measurements made with a commercial focimeter along the same lines.

(Humphrey Lens Analyzer Model 360, \pm 0.1 Dioptries precision) along the same lines.

\section{Conclusions}

We have proposed a robust procedure based on the RPT technique to demodulate squared-grating deflectograms. The RPT has been improved with the application of a more robust minimization algorithm and a quality-map-based propagation algorithm that takes into account the local reliability of the pixels in the image. Owing to the good noise rejection characteristics and the low sensitivity to the boundaries of the RPT technique, we have improved the results, compared with other techniques, to retrieve the power maps of ophthalmic lenses; furthermore, as in previously reported studies, we obtain the complete deflection information from a single deflectogram, thus avoiding the necessity of rotating the gratings. The retrieved phase is already unwrapped and is thus a good alternative for use in other applications of 
moiré deflectometry. The presented method may also be useful in techniques for which phase and gradient computations are required, for example, in experimental mechanics.

We acknowledge the support of a postdoctoral grant from the Universidad Complutense de Madrid, the "Becas Internacionales Flores-Valles" program, Spain, and the Consejo Nacional de Ciencia y Tecnología (CONACyT), México.

\section{References}

1. A. W. Lohmann and D. E. Silva, "An interferometer based on talbot effect," Opt. Commun. 2, 413-415 (1971).

2. O. Kafri and I. Glatt, The Physics of Moiré Metrology (Wiley, New York, 1989).

3. O. Kafri and A Livnat, "Reflective surface analysis using moiré deflectometry," Appl. Opt. 26, 2507-2508 (1987).

4. Z. Karny and O. Kafri, "Refractive-index measurement by moiré deflectometry,” Appl. Opt. 21, 3326-3328 (1982).

5. E. Keren and E. Bar-Ziv, "Measurements of temperature distribution flames by moiré deflectometry," Appl. Opt. 20, 42634266 (1981).

6. M. Servín and R Rodriguez-Vera, "Automatic fringe detection algorithm used for moiré deflectometry," Appl. Opt. 29, 32663270 (1990).

7. H. Canabal and J. A. Quiroga, "Automatic processing in moiré deflectometry by local fringe direction calculation," Appl. Opt. 37, 5894-5901 (1998).

8. T. Pfeifer and B. Wang, "Phase-shifting moiré deflectometry," Optik 98, 158-162 (1995).

9. H. Canabal, J. A. Quiroga, and E. Bernabeu, "Improved phaseshifting method for automatic processing of moiré deflectograms," Appl. Opt. 37, 6227-6233 (1998).

10. E. Keren and O. Kafri, "Diffraction effects in moiré deflectometry," J. Opt. Soc. Am. A 2, 111-120 (1985).

11. E. Bar-Ziv, "Effect of diffraction on the moiré image," J. Opt. Soc. Am. A 2, 371-379 (1985).
12. M. Takeda, H. Ina, and S. Kobayashi, "Fourier-transform method of fringe-pattern analysis for computer-based topography and interferometry," J. Opt. Soc. Am. A 72, 156-160 (1982).

13. M. Takeda and S. Kobayashi, "Lateral aberration measurements with a digital Talbot interferometer," Appl. Opt. 23, 1760-1764 (1984).

14. J. A. Quiroga, D. Crespo, and E. Bernabeu, "Fourier transform method for automatic processing of moiré deflectograms," Opt. Eng. 38, 974-982 (1999).

15. C. Roddier and F. Roddier, "Interferogram analysis using Fourier transform techniques," Appl. Opt. 26, 1668-1673 (1987).

16. J. Gu and F. Chen, "Fast Fourier transform, iteration, and least-squares-fit demodulation image processing of singlecarrier fringe pattern,” J. Opt. Soc. Am. A 12, 2159-2164 (1995).

17. M. Servín, J. L. Marroquín, and F. J. Cuevas, "Demodulation of a single interferogram by use of a two-dimensional regularized phase-tracking technique," Appl. Opt. 36, 4540-4548 (1997).

18. J. C. Wyant, "Double frequency grating lateral shear interferometer," Appl. Opt. 12, 2057-2060 (1973).

19. J. M. Huntley and J. E. Field, "High resolution moiré photography: application to dynamic stress analysis," Opt. Eng. 28, 926-933 (1989).

20. M. Bertero and P. Boccacci, Introduction to Inverse Problems in Imaging (Institute of Physics, London,1998), Chap. 5.

21. Matlab Optimization Toolbox, User's Guide, Version 5 (MathWorks Inc., 24 Prime Park Way, Natick, Mass., 01760-1500).

22. B. Ströbel, "Processing of interferometric phase maps as complex-valued phasor images," Appl. Opt. 35, 2192-2198 (1996).

23. R. L. Burden and J. D. Faires, Numerical Analysis, 6th ed. (International Thomson Publishing, Stamford, Conn., 1997).

24. J. Alonso, J. A. Gomez-Pedrero, and E. Bernabeu, "Local dioptric power matrix in a progressive addition lens," Ophthalmic Physiol. Opt. 17, 522-529 (1997) 